\title{
A CLASSIFICATION OF THE CUBIC SEMISYMMETRIC GRAPHS OF ORDER $34 p^{2}$
}

\author{
Mehdi Alaeiyan ${ }^{1}$ and Rasul Aramian ${ }^{2}$ \\ ${ }^{1}$ Department of Mathematics, Iran University of Science and Technology, \\ Narmak, Tehran 16844, Iran, alaeiyan@iust.ac.ir \\ ${ }^{2}$ Department of Mathematics, Iran University of Science and Technology, \\ Narmak, Tehran 16844, Iran, rasul.aramian@googlemail.com
}

\begin{abstract}
A simple undirected graph is called semisymmetric if it is regular and edge transitive but not vertex-transitive. In this paper, we classify all connected cubic semisymmetric graph of order $34 p^{2}$, where $p$ be a prime.

Key words: Symmetric graph, semisymmetric graph, regular coverings.
\end{abstract}

\begin{abstract}
Abstrak. Suatu graf tak berarah sederhana disebut semisimetris jika graf tersebut regular dan transitif sisi tapi tidak transitif titik. Dalam paper ini, kami mengklasifikasikan semua graf semisimetris kubik terhubung berorde $34 p^{2}$ dengan $p$ adalah bilangan prima.

Key words: Graf simetris, graf semisimetris, selimut regular.
\end{abstract}

\section{Introduction}

Throughout this paper, graphs are assumed to be finite, simple, undirected and connected. For a graph $X$, we use $V(X), E(X), A(X)$ and $\operatorname{Aut}(X)$ to denote its vertex set, the edge set, the arc set and the full automorphism group of $X$, respectively. For $u \in V(X), N_{X}(u)$ is the set of vertices adjacent to $u$ in $X$. For a graph $X$ and a subgroup $G$ of $\operatorname{Aut}(X), X$ is said to be $G$-vertex-transitive or $G$-edge-transitive if $G$ is transitive on the sets of vertices or edges of $X$ respectively. A graph is $G$-semisymmetric if it is $G$-vertex-transitive but not $G$-edge-transitive. Furthermore, a graph $X$ is said to be vertex-transitive or edge-transitive if in the above definition, $G=\operatorname{Aut}(X)$. It can be shown that a $G$-edge-transitive but not $G$-vertex-transitive graph is necessarily bipartite, where the two partite parts of

2000 Mathematics Subject Classification: 05C10, $20 \mathrm{~B} 25$.

Received: 11-11-2010, revised: 28-02-2011, accepted: 04-03-2011. 
the graph are orbits of $G$. Moreover, if $X$ is regular these two partite sets have equal cardinality. A regular edge- but not vertex-transitive graph will be referred to as a semisymmetric graph.

The class of semisymmetric graphs was first introduced by Folkman [4], and Malnič et al. [7] classified cubic semisymmetric graphs of order $2 p^{3}$ for a prime $p$, while Folkman [4] proved that there is no cubic semisymmetric graphs of order $2 p$ or $2 p^{2}$. In this paper, we show that there are no connected cubic semisymmetric graphs of order $34 p^{2}$.

Let $X$ be a graph and $N$ a subgroup of $\operatorname{Aut}(X)$. Denote by $X_{N}$ the quotient graph corresponding to the orbits of $N$, that is the graph having the orbits of $N$ as vertices with two orbits adjacent in $X_{N}$ whenever there is an edge between those orbits in $X$. A graph $\widetilde{X}$ is called a covering of a graph $X$ with projection $\wp: \widetilde{X} \rightarrow X$ if there is a surjection $\wp: V(\widetilde{X}) \rightarrow V(X)$ such that $\left.\wp\right|_{N_{\widetilde{X}}(\tilde{v})}: N_{\widetilde{X}}(\tilde{v}) \rightarrow N_{X}(v)$ is a bijection for any vertex $v \in V(X)$ and $\tilde{v} \in \wp^{-1}(v)$. A covering $\tilde{X}$ of $X$ with a projection $\wp$ is said to be regular (or $K$-covering) if there is a semiregular subgroup $K$ of the automorphism group $\operatorname{Aut}(\widetilde{X})$ such that graph $X$ is isomorphic to the quotient graph $\widetilde{X}_{K}$, say by $h$, and the quotient map $\widetilde{X} \rightarrow \widetilde{X}_{K}$ is the composition $\wp h$ of $\wp$ and $h$.

Proposition 2.1. (1) [9, Theorem 8.5.3] Let $p$ and $q$ be primes and let $a$ and $b$ be non-negative integers. Then every group of order $p^{a} p^{b}$ is solvable.

(2) [3, Feit-Thompson Theorem] Every finite group of odd order is solvable.

Proposition 2.2. [10, p. 236] Let $G$ be a finite group. If $G$ has an abelian Sylow p-subgroup, then $p$ does not divide $\left|G^{\prime} \cap Z(G)\right|$.

Proposition 2.3. [5] The vertex stabilizers of a G-semisymmetric cubic graph has order $2^{r}$. 3 , where $0 \leq r \leq 7$.

The next preposition is special case of [8, Lemma 3.2].

Proposition 2.4. Let $X$ be a connected $G$-semisymmetric cubic with bipartition sets then $U(X)$ and $W(X)$, and let $N$ be a normal subgroup of $G$. If $N$ is intransitive on bipartition sets then $N$ acts semiregularly on both $U(X)$ and $W(X)$, and $X$ is an $N$-covering of a $G / N$-semirsymmetric graph.

\section{Main Results}

The following is the main result of this paper.

Theorem 2.1. Let $p$ be a prime. Then there is no connected cubic semisymmetric graph of order $34 p^{2}$.

Proof. For $p \leq 3$, by [1], there is no connected cubic semisymmetric graph of order $34 p^{2}$. Thus we may assume that $p \geq 5$. To prove the theorem, we only need 
to show that no connected cubic semisymmetric graph of order $34 p^{2}$ exits for $p \geq 5$. Suppose to the contrary that $X$ is such a graph. Since semisymmetric graphs are bipartite, one may denote by $U(X)$ and $W(X)$ the bipartite sets of $X$. Clearly, $|U(X)|=|W(X)|=17 p^{2}$. Set $A:=\operatorname{Aut}(X)$. By proposition 2.3, $|A|=2^{r}$. 3. 17. $p^{2}$ for some integer $0 \leq r \leq 7$. Let $N$ be a minimal normal subgroup of $A$.

Assume that $N$ is unsolvable. Then $N$ is a product of isomorphic non-abelian simple groups and hence a non-abelian simple group because $|A|=2^{s}$. 3. 17. $p^{2}$. Suppose that $N$ is not transitive on bipartition sets $U(X)$ and $W(X)$. By Proposition 2.4, $N$ acts semiregularly on bipartition sets $U(X)$ and $W(X)$. Thus, $|N| \mid$ $34 p^{2}$. This forces that $N$ is solvable, a contradiction. It follows that $N$ is transitive on at least one of the bipartition sets $U(X)$ and $W(X)$, implying $17 p^{2}|| N \mid$. By Proposition 2.1, $|N|=2^{t}$. 17. $p^{2}$ or $2^{t}$. 3. 17. $p^{2}$. Let $q$ be a prime. Then by [6, pp. $12-14]$ and [2], a non-abelian simple $\{2, q, p\}$-group is one of the following groups

$$
A_{5}, A_{6}, P S L_{2}(7), P S L_{2}(8), P S L_{2}(17), P S L_{3}(3), P S U_{3}(3) \text { and } P S U_{4}(2) \text {, }
$$

With orders $2^{2} .3 .5,2^{3} .3^{2} .5,2^{3} .3 .7,2^{3} .3^{2} .7,2^{4} .3^{2} .17,2^{4} \cdot 3^{3} \cdot 13,2^{5} .3^{3} .7$ and $2^{6} .3^{4} .5$, respectively. This implies that for $p \geq 5$, there is no simple group of order $2^{t}$. 17. $p^{2}$. Hence $|N|=2^{t}$. 3. 17. $p^{2}$.

Assume that $L$ is a proper subgroup of $N$. If $L$ is unsolvable, then $L$ has a non-abelian simple composite factor $L_{1} / L_{2}$. Since $p \geq 5$ and $\left|L_{1} / L_{2}\right| \mid 2^{t}$. 3. 17. $p^{2}$, by simple group listed in (1), $L_{1} / L_{2}$ cannot be a $\{2,3,17\}$ - or $\{2,17, p\}$-group. If $L_{1} / L_{2}$ is $\{2,3, p\}$-group, then $p=5$ or 7 and hence $N$ is $A_{5}$ or $P S L_{2}(7)$, which is impossible, because by [2, pp. 239], there is no non-abelian simple group of order $|N|=2^{t}$. 3. 17. $p^{2}$ for $p=5,7$. Thus, $L_{1} / L_{2}$ is a $\{2,3,17, p\}$ group. One may assume that $|L|=2^{s}$. 3. 17. $p^{2}$ or $2^{s}$. 3. 17. $p$, where $s \geq 2$. Let $|L|=2^{s}$. 3. 17. $p^{2}$. Since $N=2^{t}$. 3. 17. $p^{2}$ for some $0 \leq t \leq 7$, one has $|N: L| \leq 32$. Consider the action of $N$ on the right cosets of $L$ in $N$ by right multiplication. The simplicity of $N$ implies that this action is faithful. It follows $N \leq S_{32}$ and hence $p=5,7,11,13,17,19,23,29$ or 31 . But by [2, pp. 239], there is no non-abelian simple group of order $|N|=2^{t}$. 3. 17. $p^{2}$ for $p=5,7,11,13,17,19,23,29,31$, a contradiction. Thus, $L$ is solvable and hence $N$ is a minimal non-abelian simple group, that is, $N$ is a non-abelian simple group and every proper subgroup of $N$ is solvable. By [11, Corollary 1], $N$ is one of the groups in Table I. It can be easily verified that the order of groups in Table I are not of the form $2^{t}$. 3. 17. $p^{2}$.

Thus $|L|=2^{s}$. 3. 17. $p$. By the same argument as in the preceding paragraph (replacing $N$ by $L$ ) $L$ is one of the groups in Table I. Since $|L|=2^{s}$. 3. 17. $p$, the possible candidates for $L$ is $P S L_{2}(m)$. Clearly, $m=p$. We show that $|L| \leq$ $2,500,224$. If $17 \nmid(p-1) / 2$, then $(p-1) / 2 \mid 2^{7}$. 3, which implies that $p \leq 769$. If $p=769$, then $2^{8}|| L \mid$, a contradiction. Thus $p<769$ and hence $p \leq 193$ because $(p-1) / 2 \mid 2^{7}$. 3. It follows that $|L| \leq 2^{7}$. 3. 17. $193=1,259,904<$ $2,500,224$. If $17 \mid(p-1) / 2$, Then $p+1 \mid 2^{7}$. 3. Consequently $p \leq 383$, implying $|L| \leq 2^{7}$. 3. 17. $383 \leq 2,500,224$. Thus, $|L| \leq 2,500,224$. Then by [2, pp. 239], is isomorphic to $P S L_{2}(16)$. It follows that $p=5$ and hence $|N|=2^{t} .3 .17 .5^{2}$, which is impossible by [2, pp. 239]. 
Table I. The possible for non-abelian simple group $N$.

\begin{tabular}{ll}
\hline$N$ & $|N|$ \\
\hline$P S L(2, m), m>3$ a prime and $m^{2} \neq 3(\bmod 5)$ & $\frac{1}{2} m(m-1)(m+1)$ \\
$P S L\left(2,2^{n}\right), n$ a prime & $2^{n}\left(2^{2 n}-1\right)$ \\
$P S L\left(2,3^{n}\right), n$ an odd prime & $\frac{1}{2} 3^{n}\left(3^{2 n}-1\right)$ \\
$P S L(3,3), n$ a prime & $13.3^{3} \cdot 2^{4}$ \\
Suzuki group $S z\left(2^{n}\right), n$ an odd prime & $2^{2 n}\left(2^{2 n}+1\right)\left(2^{n}-1\right)$ \\
\hline
\end{tabular}

Thus $N$ is solvable and hence elementary abelian. Therefore $N$ is intransitive on both $U(X)$ and $W(X)$ and by Proposition 2.4,N is semiregular on $U(X)$ and $W(X)$. Set $Q:=O_{p}(A)$. If $|Q|=p^{2}$, then by Proposition 2.4, the quotient graph $X_{Q}$ of $X$ corresponding to the orbits of $Q$ is a $A / Q$-semisymmetric graph of order 34 , which is impossible by [1]. Thus $|Q|=1$ or $p$. Suppose first that $Q=1$. Now we consider the quotient graph $X_{N}$ of $X$ corresponding to the orbits of $N$. The semiregularity of $N$ implies that $N \cong \mathbb{Z}_{17}$, because $Q=1$. By Proposition $2.4 X_{N}$ is $A / N$-semisymmetric. We denote by $U\left(X_{N}\right)$ and $W\left(X_{N}\right)$ the bipartition sets of $X_{N}$. Clearly, $\left|U\left(X_{N}\right)\right|=\left|W\left(X_{N}\right)\right|=p^{2}$. Let $L / N$ be a minimal normal subgroup of $A / N$. By the same argument as above we may prove that $L / N$ is solvable and hence elementary abelian, which implies that $L / N$ is intransitive on bipartite sets of $X_{N}$. Then by Proposition $2.4, L / N$ is semiregular on $U\left(X_{N}\right)$ and $W\left(X_{N}\right)$, implying $|L / N| \mid p^{2}$. Hence $L / N \cong \mathbb{Z}_{p}$ or $\mathbb{Z}_{p}^{2}$. Thus $|L|=17 p$ or $17 p^{2}$. Since $p \geq 5$, by Sylow's theorem $L$ has a normal subgroup of order $p$ or $p^{2}$, which is characteristic in $L$ and hence is normal in $A$, because $L \triangleleft A$. This contradicts our assumption that $Q=1$.

Suppose now that $Q \cong \mathbb{Z}_{p}$. Let $C:=C_{A}(Q)$ be the centralizer of $Q$ in $A$. By Proposition 2.2, $p \nmid\left|C^{\prime} \cap Z(C)\right|$ and hence $C^{\prime} \cap Q=1$, where $C^{\prime}$ is the derived subgroup of $C$. This forces $p^{2} \nmid\left|C^{\prime}\right|$, because $C^{\prime}$ is normal in $A$. It follows that $C^{\prime}$ is intransitive on $U(X)$ and $W(X)$. As $C^{\prime}$ is normal in $A$, by Proposition 2.4, it is semiregular on $U(X)$ and $W(X)$. Moreover, the quotient graph $X_{C^{\prime}}$ is $A / C^{\prime}$ semisymmetric and since $p^{2} \nmid\left|C^{\prime}\right|$, the semiregularity of $C^{\prime}$ implies that $\left|C^{\prime}\right| \mid 17 p$. Since the Sylow $p$-subgroups of $A$ are abelian, one has $p^{2}|| C \mid$ and so $p|| C / C^{\prime} \mid$. Now let $K / C^{\prime}$ be a Sylow $p$-subgroup of the abelian group $C / C^{\prime}$. As $K / C^{\prime}$ is characteristic in $C / C^{\prime}$ and $C / C^{\prime} \triangleleft A / C^{\prime}$, we have that $K / C^{\prime} \triangleleft A / C^{\prime}$. Hence $K$ is normal in $A$. Clearly $|K|=17 p^{2}$ because $|Q|=p$. Since $p \geq 5, K$ has a normal subgroup of order $p^{2}$, which is characteristic in $K$ and hence is normal in $A$, contradicting to $Q \cong \mathbb{Z}_{p}$.

\section{References}

[1] Conder, M., Malnič, A., Marušič, D. and Potočnik, P., "A census of cemisymmetric cubic craphs on up to 768 vertices", J. Algebraic Combin. 23 (2006), 255-294.

[2] Conway, J. H., Curties, R. T., Norton, S. P., Parker, R. A. and Wilson, R. A., Atlas of Finite Groups, Clarendon Press, Oxford, 1985.

[3] Feit, W. and Thompson, J. G., "Solvability of groups of odd order", Pacific J. Math. 13 (1936), 775-1029. 
[4] Folkman, J., "Regular line-symmetric graphs", J. Combin Theory 3 (1967) 215-232.

5] Goldschmidt, D., "Automorphisms of trivalent graphs", Ann. Math. 111 (1980) 377406

[6] Gorenstein, D., Finite Simple Groups, Plenum Press, New York, 1982.

[7] Malnič, A., Marušič, D. and Wang, C.Q., "Cubic edge-transitive graphs of order $2 p^{3}$ ", Discrete Math. 274 (2004) 187-198.

[8] Lu, Z., Wang, C.Q. and Xu, M. Y., On semisymmetric cubic graphs of order $6 p^{2}$, Science in Chaina Ser. A Mathematics 2004 Vol. 47 No.11-17.

[9] Robinson, D. J., A Course On Group Theory, Cambridge University Press, 1978.

[10] Rose, J. S., A Course in the theory of groups, Springer-Verlage, Berlin, 1979.

[11] Thompson, J. G., "Nonsolvable finite groups all of whose local subgroups are solvable: I", Bull amer Math Soc 74 (1967), 383-435. 